03

\title{
О сфероидальной модели рассеяния света несферическими частицами
}

\author{
() В.Г. Фарафронов ${ }^{1}$, В.Б. Ильин ${ }^{1,2,3}$, М.С. Прокопьева ${ }^{2}$, А.Р. Тулегенов ${ }^{1}$, В.И. Устимов ${ }^{1}$ \\ ${ }^{1}$ Государственный университет аэрокосмического приборостроения, \\ 190000 Санкт-Петербург, Россия \\ ${ }^{2}$ Санкт-Петербургский государственный университет, \\ 199034 Санкт-Петербург, Россия \\ ${ }^{3}$ Главная (Пулковская) астрономическая обсерватория РАН, \\ 196140 Санкт-Петербург, Россия \\ e-mail: far@aanet.ru
}

Поступило в редакцию 30.11.2018 г.

В окончательной редакции 30.11.2018 г.

Принята к печати 11.12.2018 г.

Построена сфероидальная модель для решения проблемы рассеяния света несферическими частицами. Полуоси модельного сфероида определяются исходя из равенства объемов, а также равенства отношений продольных и поперечных размеров исходной частицы и модели, что обеспечивает близость их оптических свойств. Данный подход был применен к вытянутым и сплюснутым параллелепипедам, цилиндрам и конусам с отношениями большего размера к меньшему, равными 2 и 10. Направление распространения падающей плоской волны ТЕ- или ТМ-типа было либо параллельным, либо перпендикулярным к оси симметрии частиц и модельного сфероида. Размер частиц определялся безразмерным параметром $x_{v}=2 \pi r_{v} / \lambda$, который зависит от объема частицы, так как $r_{v}$ представляет собой радиус эквиобъемного шара. При расчетах данный параметр менялся от малых величин до достаточно больших $x_{v}=10$. Область применимости модели определялась сравнением результатов численных расчетов по строгим методам разделения переменных для сфероидов и дискретных диполей для других несферических частиц. Показано, что область применимости модели для параллелепипедов, цилиндров и конусов достаточно широка для разных параметров задачи, в частности, если параметр $x_{v} \leq 6$, то относительная погрешность модели не превышает $10-15 \%$. В значительной степени это связано с тем фактом, что первый максимум зависимости факторов рассеяния $Q_{\text {sca }}$ от $x_{v}$ оказывается сходным для частиц разной формы, аппроксимируемых одним модельным сфероидом.

DOI: $10.21883 /$ OS.2019.04.47514.345-18

\section{Введение}

Рассеяние света несферическими частицами являются важной частью фундаментальных и прикладных проблем в оптике атмосферы, медицине, астрофизике, биофизике и других областях науки и техники [1-3]. Методы решения подобных задач сегодня интенсивно развиваются различными научными группами [4-7]. Несмотря на достигнутый прогресс в разработке методов, многочисленные приложения, особенно связанные с решением обратных задач при зондировании дисперсных сред, требуют применения простых, но физически обоснованных моделей натуральных и антропогенных рассеивателей для ускорения расчетов оптических свойств последних.

Наиболее привлекательной моделью несферической частицы является сфероид (эллипсоид вращения). В рамках этой модели можно рассматривать как вытянутые, так и сплюснутые частицы с произвольной степенью асферичности. Для сфероидальных частиц разработаны как точные, так и приближенные методы. Из первых наиболее известными являются метод разделения переменных (separation of variables method, SVM), метод расширенных граничных условий (extended boundary condition method, EBCM) и метод дискретных диполей (discrete dipole approximation, DDA) (см. описание всех трех методов, например, в соответствующих главах [2]). Первый из них является наиболее эффективным, так как наиболее полно учитывает геометрию задачи за счет использования сфероидального базиса, т.е. представления полей в виде рядов по векторным сфероидальным волновым гармоникам. К недостатком данного метода можно отнести трудности, связанные с численными расчетами сфероидальных функций в широком диапазоне изменения параметров. Второй метод основывается на разложении полей по векторным сферическим гармоникам, что естественно затрудняет его применение к сфероидам с большим отношением полуосей. Последний подход может быть использован для решения задачи рассеяния света частицами произвольной формы, однако такая универсальность соответственно требует больших (иногда невозможных) затрат оперативной памяти и процессорного времени.

Сфероидальная модель неоднократно применялась для моделирования оптических свойств различных полидисперсных сред, в частности частиц над пустыней [8], минеральной пыли [9], частиц в перистых облаках [10], ледяных гидрометеоров [11] и т. п. Широкое распространение модели в основном объясняется тем, что она 
является простой и достаточно гибкой (см., например, обсуждение в [12]). Основания для применения сфероидальной модели обсуждались в частном случае рассеяния света частицами, малыми по сравнению с длиной волны падающего излучения (например, $[13,14])$. При этом был сделан вывод о том, что умеренные отклонения (даже нарушающие гладкость) формы частиц от сферической играют меньшую роль, чем изменения отношения наибольшей протяженности к наименьшей. Этот вывод был недавно подтвержден с использованием иного подхода к расчету поляризуемости и для других форм малых рассеивателей в работах $[15,16]$.

В настоящей работе рассматривается применимость сфероидальной модели для определения оптических свойств нескольких видов несфероидальных частиц (параллелепипедов, цилиндров и конусов), размер которых больше или сравним с длиной волны падающего излучения. В разд. 1 и 2 кратко описываются точные методы, использованные нами для моделирования рассеяния света сфероидальными и несфероидальными частицами. В разд. 3 приведены и обсуждаются результаты численных расчетов сечения рассеяния для частиц разной формы и точность апроксимации этих сечений при использовании сфероидальной модели.

\section{1. Метод разделения переменных}

Метод разделения переменных был впервые применен для решения задачи рассеяния света сфероидальными частицами Асано и Ямамото [17]. Позднее более эффективная схема подобного подхода была реализована Фарафоновым и Вощинниковым $[18,19]$.

Сфероид получается путем вращения эллипса вокруг одной из своих осей. Форма сфероида определяется отношением $a / b$, где $a$ и $b-$ большая и малая полуоси. Для определения размера частицы можно использовать безразмерный параметр

$$
x_{v}=\frac{2 \pi r_{v}}{\lambda}=k r_{v},
$$

где $\lambda$ - длина волны излучения, $k=2 \pi / \lambda-$ волновое число, $r_{v}$ - радиус сферы, объем которой равен объему сфероида. Для вытянутой сфероидальной частицы имеем

$$
r_{v}^{3}=a b^{2} .
$$

Аналогично линейный дифракционный параметр записывается в виде

$$
x_{a}=\frac{2 \pi a}{\lambda}=k a .
$$

Для сплюснутых сфероидов в приведенных выше формулах полуоси $a$ и $b$ следует поменять местами.

Оптические свойства вытянутых сфероидов будем описывать с помощью факторов

$$
Q=\frac{C}{\pi r_{v}^{2}}
$$

где $C$ - сечения ослабления или рассеяния.
Сфероидальная система координат $(\xi, \eta, \varphi)$ связана с декартовой системой координат $(x, y, z)$ следующими соотношениями:

$$
\begin{gathered}
x=\frac{d}{2}\left(\xi^{2} \mp 1\right)^{1 / 2}\left(1-\eta^{2}\right)^{1 / 2} \cos \varphi, \\
y=\frac{d}{2}\left(\xi^{2} \mp 1\right)^{1 / 2}\left(1-\eta^{2}\right)^{1 / 2} \sin \varphi, \\
z=\frac{d}{2} \xi \eta,
\end{gathered}
$$

где для вытянутой системы координат $\xi \in[1, \infty)$, $\eta \in[-1,1], \varphi \in[0,2 \pi)$ (верхний знак), для сплюснутой системы координат $\xi \in[0, \infty), \eta \in[-1,1], \varphi \in[0,2 \pi)$ (нижний знак), $d-$ фокусное расстояние сфероида.

Для вытянутых сфероидальных частиц факторы ослабления записываются в виде [19]

$$
\begin{aligned}
Q_{\mathrm{ext}}= & \frac{4}{x_{v}^{2}} \operatorname{Re}\left\{-\sum_{l=1}^{\infty} i^{-l} a_{l}^{(1)} S_{1 l}(c, \cos \alpha)\right. \\
& +\sum_{m=1}^{\infty} \sum_{m=l}^{\infty} i^{-(l-1)}\left(k a_{m l}^{(1)} S_{m l}(c, \cos \alpha)\right. \\
& \left.\left.+i b_{m l}^{(1)} S_{m l}^{\prime}(c, \cos \alpha)\right) \sin \alpha\right\},
\end{aligned}
$$

где $c=k d / 2, S_{m l}(c, \eta)$ - вытянутые угловые сфероидальные функции с коэффициентами нормализации $N_{m l}(c), \xi_{0}$ - значение радиальной координаты, соответствующей поверхности сфероида, штрих означает дифференцирование, $\alpha$ - угол между осью вращения сфероида и направлением падающего излучения. Коэффициенты разложения рассеянного поля по сфероидальному базису $a_{m l}^{(1)}$ и $b_{m l}^{(1)}$ определяются из бесконечных систем линейных алгебраических уравнений (см. подробнее [19]).

Аналогично для факторов рассеяния имеем

$$
\begin{aligned}
Q_{\text {sca }}= & \frac{1}{x_{v}^{2}}\left\{2 \sum_{l=1}^{\infty}\left|a_{l}^{(1)}\right|^{2} N_{1 l}^{2}(c)\right. \\
& +\operatorname{Re} \sum_{l=1}^{\infty} \sum_{l=m}^{\infty} \sum_{n=m}^{\infty} i^{n-l}\left[k^{2} a_{m l}^{(1)} a_{m n}^{(1) *} \omega_{l n}^{(m)}(c, c)\right. \\
& +i k\left(b_{m l}^{(1)} a_{m n}^{(1) *} \kappa_{l n}^{m}(c, c)-a_{m l}^{(1)} b_{m n}^{(1) *} \kappa_{n l}^{m}(c, c)\right) \\
& \left.\left.+b_{m l}^{(1)} b_{m n}^{(1) *} \tau_{l n}^{(m)}(c, c)\right] N_{m l}(c) N_{m n}(c)\right\},
\end{aligned}
$$

где $\omega_{l n}^{(m)}$ и $\tau_{l n}^{(m)}$ - интегралы от угловых сфероидальных функций, а звездочкой отмечены сопряженные значения.

\section{2. Метод дискретных диполей}

Метод DDA был разработан Парселем и Пеннипакером [20] как способ расчета рассеяния и поглощения 
света частицами произвольной формы с размерами, сравнимыми или меньшими длины волны. Подход заключается в предствлении твердой частицы набором точечных диполей, расстояние между которыми мало по сравнению с длиной волны. Каждый диполь обладает поляризуемостью в отклике к падающей плоской волне и электрическим полям, образуемым остальными диполями частицы. Диполи обычно размещаются на простой кубической решетке [21].

Итак, частица заменяется набором из $N$ диполей с координатами $\mathbf{r}_{i}$ и поляризуемостями $\alpha_{i}$. Рассеяние и поглощение диполями может быть рассчитано с высокой точностью. Поляризуемость в точке $\mathbf{r}_{i}$ вычисляется следующим образом:

$$
\mathbf{P}_{i}=\alpha_{i} \mathbf{E}\left(\mathbf{r}_{i}\right),
$$

где $\mathbf{r}_{i}-$ положение диполя с поляризуемостью $\alpha_{i}$. Поле в правой части $\mathbf{E}\left(\mathbf{r}_{i}\right)$ представляет собой сумму падающей волны

$$
\mathbf{E}_{\text {inc }, i}=\mathbf{E}_{0} \exp \left(i \mathbf{k} \mathbf{r}_{i}-i \omega t\right)
$$

и волн, создаваемых другими диполями,

$$
\mathbf{E}_{\mathrm{self}, i}=-\sum_{j \neq i} A_{i j} \mathbf{P}_{j}
$$

В результате уравнение (8) может быть записано следующим образом:

$$
\left(\alpha_{i}\right)^{-1} \mathbf{P}_{i}+\sum_{j \neq i} A_{i j} \mathbf{P}_{j}=\mathbf{E}_{\mathrm{inc}, i} .
$$

Определение $A_{i i}=\left(\alpha_{i}\right)^{-1}$ упрощает решение проблемы рассеяния и нахождение поляризуемости $\mathbf{P}_{i}$, которая удовлетворяет системе $3 N$ комплексных линейных уравнений:

$$
\sum_{k=1}^{N} A_{i j} \mathbf{P}_{i}=\mathbf{E}_{\text {inc }, i}
$$

После решения системы (12) сечения экстинкции $C_{\text {ext }}$ и поглощения $C_{\text {abs }}$ рассчитываются по формулам [22]

$$
\begin{gathered}
C_{\mathrm{ext}}=\frac{4 \pi k}{\left|\mathbf{E}_{0}\right|^{2}} \sum_{i=1}^{N} \operatorname{Im}\left(\mathbf{E}_{\mathrm{inc}, i}^{*} \mathbf{P}_{i}\right), \\
\left.C_{\mathrm{abs}}=\frac{4 \pi k}{\left|\mathbf{E}_{0}\right|^{2}} \sum_{i=1}^{N}\left\{\operatorname{Im}\left[\mathbf{P}_{i} \cdot\left(\alpha_{i}^{-1}\right)^{*} \mathbf{P}_{i}^{*}\right)\right]-\frac{2}{3} k^{3}\left|\mathbf{P}_{i}\right|^{2}\right\} .
\end{gathered}
$$

Наконец, сечение рассеяния определяется из закона сохранения энергии: $C_{\mathrm{sca}}=C_{\mathrm{ext}}-C_{\mathrm{abs}}$.

\section{3. Результаты численных расчетов и обсуждение}

В общем случае при построении эллипсоидальной модели мы заменяем исходную частицу неким „эффективным“ эллипсоидом. При этом полуоси $a_{\mathrm{ef}}, b_{\mathrm{ef}}$ и $c_{\mathrm{ef}}$ „эффективного“ эллипсоида вычисляются из двух соображений. Во-первых, объемы модельного эллипсоида и несферической частицы должны совпадать:

$$
V_{\mathrm{ef}}=V_{\text {part }}
$$

Во-вторых, для учета формы частицы отношения ее поперечных и продольных размеров должны быть равны отношениям соответствующих полуосей модели:

$$
\frac{a_{\mathrm{ef}}}{c_{\mathrm{ef}}}=\frac{a_{\mathrm{part}}}{c_{\mathrm{part}}}, \quad \frac{b_{\mathrm{ef}}}{c_{\mathrm{ef}}}=\frac{b_{\mathrm{part}}}{c_{\mathrm{part}}} .
$$

В случае осесимметричной частицы „эффективный“ эллипсоид превращается в вытянутый или сплюснутый „эффективный“ сфероид, что дает соотношения

$$
V_{\mathrm{ef}}=V_{\mathrm{part}}, \quad \frac{a_{\mathrm{ef}}}{b_{\mathrm{ef}}}=\frac{a_{\text {part }}}{b_{\text {part }}} .
$$

В данной работе мы применяем сфероидальную модель к прямоугольным параллепипедам, стороны основания которых равны, круговым цилиндрам конечной длины и прямым круговым конусам. При определении полуосей $a_{\text {ef }}, b_{\text {ef }}$ и $c_{\text {ef }}$ модельного эллипсоида в случае параллелепипеда со сторонами $a, b, c$ из формул (15), (16) нетрудно получить следующий результат:

$$
a_{\mathrm{ef}}=\sqrt[3]{\frac{3}{4 \pi}} a, \quad b_{\mathrm{ef}}=\sqrt[3]{\frac{3}{4 \pi}} b, \quad c_{\mathrm{ef}}=\sqrt[3]{\frac{3}{4 \pi}} c,
$$

поскольку здесь имеет место подобное изменение размеров. Для конечных цилиндров длины $L$ и диаметра $D$ вычисление полуосей „эффективного“ сфероида осуществляется по формулам

$$
a_{\mathrm{ef}}=\sqrt[3]{\frac{3}{16}} D, \quad b_{\mathrm{ef}}=\sqrt[3]{\frac{3}{16}} L .
$$

По сравнению с цилиндром конус высотой $H$ и радиусом $R$ не имеет симметрии относительно плоскости, параллельной $x y$, если его ось симметрии совпадает с осью z. „Эффективный“ сфероид строится аналогично случаю цилиндра:

$$
a_{\mathrm{ef}}=\sqrt[3]{\frac{1}{2}} R, \quad b_{\mathrm{ef}}=\sqrt[3]{\frac{1}{16}} H .
$$

Если $H=2 R$, то „эффективный“ сфероид становится шаром. Заметим, что в приведенных выше формулах рассматриваются вытянутые осесимметричные частицы, в противном случае $a_{\text {ef }}$ и $b_{\text {ef }}$ нужно поменять местами.

Численные расчеты оптических свойств сфероидов проводились нами с использованием метода SVM со сфероидальным базисом [19], а для частиц, имеющих несфероидальную форму - с примененим метода DDA. Современная версия этого метода [23] позволяет моделировать рассеяние света частицами произвольной формы, а также имеет упрощенную процедуру задания многих видов частиц. Мы рассматривали вытянутые 
и сплюснутые параллелепипеды, цилиндры и конусы с отношениями большего линейного размера к меньшему $a / b=2$ или 10 . Направление распространения падающей плоской волны ТЕ- или ТМ-типов было либо параллельным, либо перпендикулярным оси вращения модельного сфероида. Размер частиц определялся безразмерным параметром $x_{v}=2 \pi r_{v} / \lambda$, который зависит от объема частицы, так как $r_{v}$ представляет собой радиус эквиобъемного шара. При расчетах данный параметр менялся от малых величин до достаточно

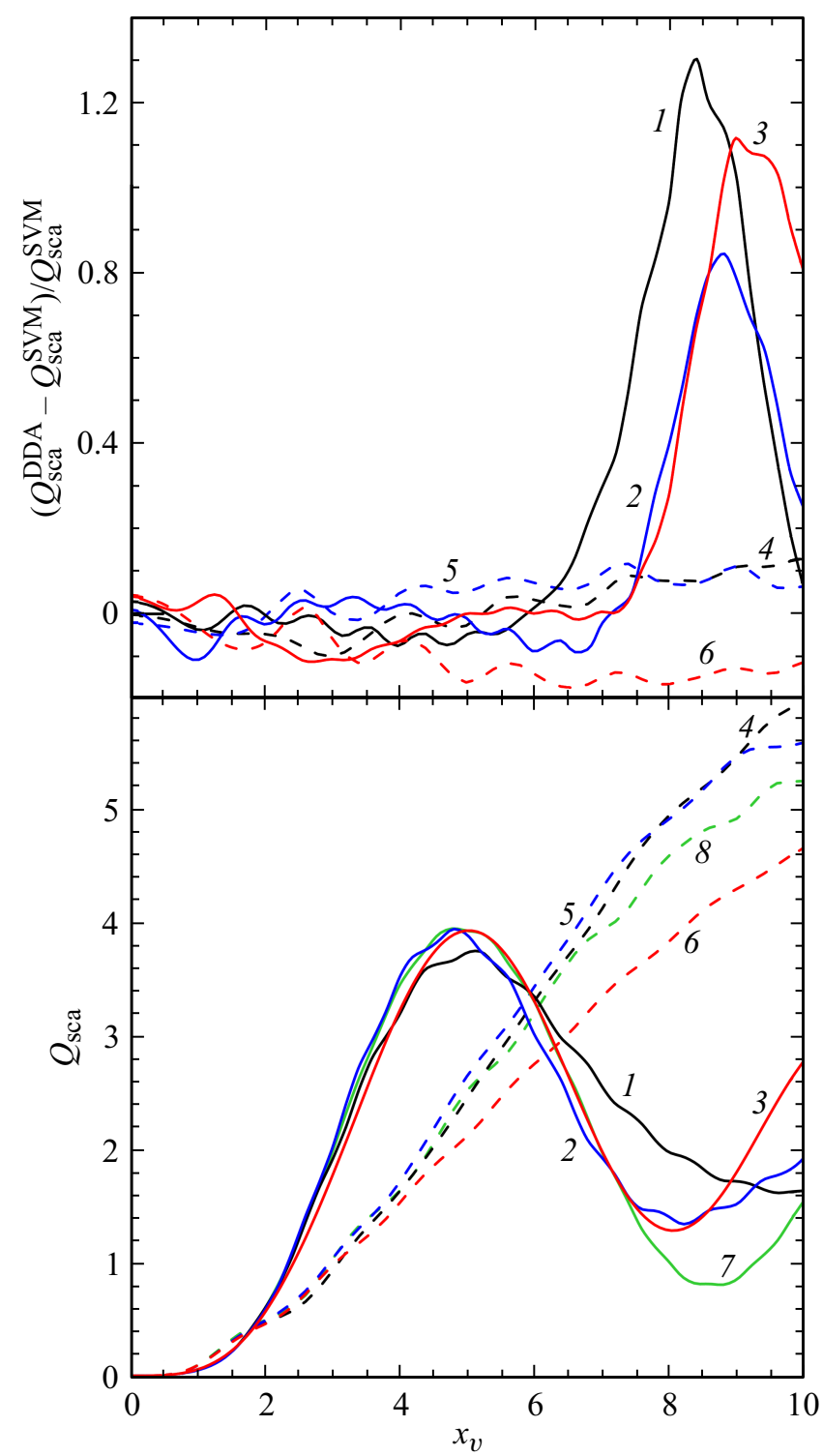

Рис. 1. Верхняя панель: погрешность факторов $Q_{\text {sca, полу- }}$ ченных с использованием сфероидальной модели для разных частиц при $m=1.3$ и $\alpha=0$, в зависимости от $x_{v}$; параллелепипеды с $a / b=2$ (сплошная кривая 1 ) и 0.5 (штриховая кривая 4); цилиндры с $L / D=2$ (сплошная кривая 2) и 0.5 (штриховая кривая 5); конусы с $H / D=2$ (сплошная кривая 3 ) и 0.5 (штриховая кривая 6). Нижняя панель: зависимость факторов $Q_{\text {sca }}$ от $x_{v}$, добавлены кривые для сфероидов с $a / b=2$ (сплошная кривая 7) и 0.5 (штриховая кривая 8 ).

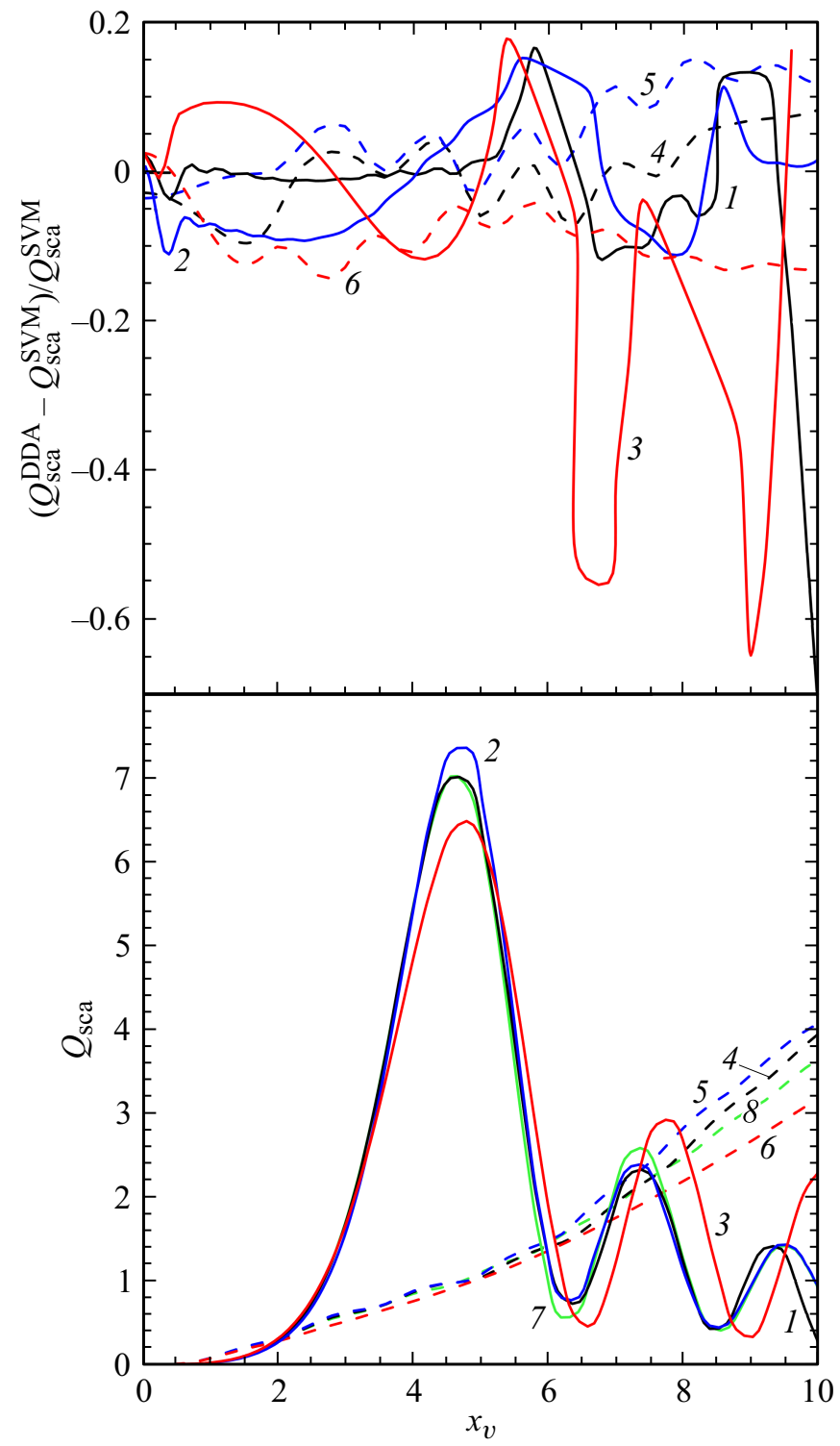

Pис. 2. То же, что и на рис. 1 , но для отношений $a / b, H / D$ и $L / D$, равных 10 (сплошные кривые) и 0.1 (штриховые кривые).

больших: $x_{v} \leq 10$. Расчеты проводились для непоглощающих частиц с показателем преломления $m=1.3$, и поэтому рассматривались только факторы рассеяния $Q_{\mathrm{sca}}$. Заметим, что линейный размер частиц определялся соответствующим безразмерным параметром $x_{a}=2 \pi a / \lambda$. Объемный дифракционный параметр $x_{v}$ и соответствующий линейный параметр $x_{a}$ связаны соотношениями $x_{a} / x_{v}=\left(a_{\mathrm{ef}} / b_{\mathrm{ef}}\right)^{1 / 3}$ для сплюснутых частиц и $x_{a} / x_{v}=\left(a_{\mathrm{ef}} / b_{\mathrm{ef}}\right)^{2 / 3}$ для вытянутых. Рассматриваемые частицы могут иметь достаточно большие продольные размеры по сравнению с длиной волны падающего излучения. Например, для сплюснутых частиц с отношением $a_{\mathrm{ef}} / b_{\mathrm{ef}}=10$ и объемным параметром $x_{v}=10$ получим линейный параметр $x_{a} \approx 21.5$, а для аналогичных вытянутых частиц уже $x_{a} \approx 46.5$. 
Очевидно, что в рамках нашей модели разные несферические частицы, имеющие одинаковый объем (15) и отношение поперечных и продольного размеров (16), соответствуют одному „эффективному“ сфероиду. В силу этого для них следует ожидать примерно одинаковых факторов эффективности рассеяния $Q_{\mathrm{sca}}^{\mathrm{DDA}}$, которые должны слабо отличаться от соответствующих факторов $Q_{\mathrm{sca}}^{\mathrm{SVM}}$ для модельных сфероидов. Относительная погрешность результатов, полученных нами для сфероидальных моделей, вычислялась следующим образом:

$$
\delta=\left|Q_{\mathrm{sca}}^{\mathrm{DDA}} / Q_{\mathrm{sca}}^{\mathrm{SVM}}-1\right|,
$$

где индекс DDA обозначает факторы несфероидальных частиц, а индекс SVM - факторы, полученные для „эффективного“ сфероида.

Результаты расчетов для рассмотренных несфероидальных частиц приведены на рис. 1-6. На каждом

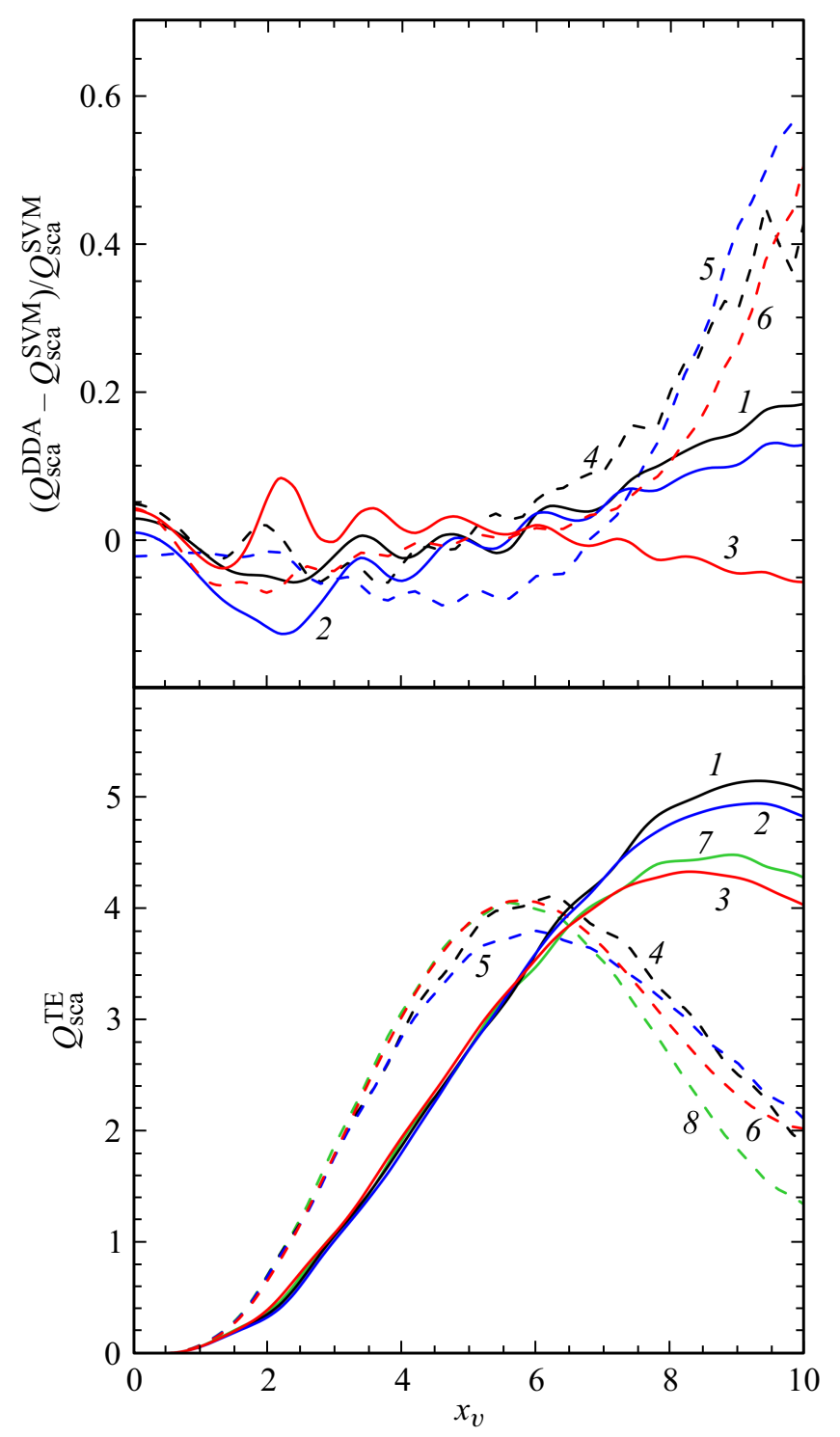

Pис. 3. То же, что и на рис. 1 , но для угла падения $\alpha=90^{\circ}$ и ТЕ-моды.

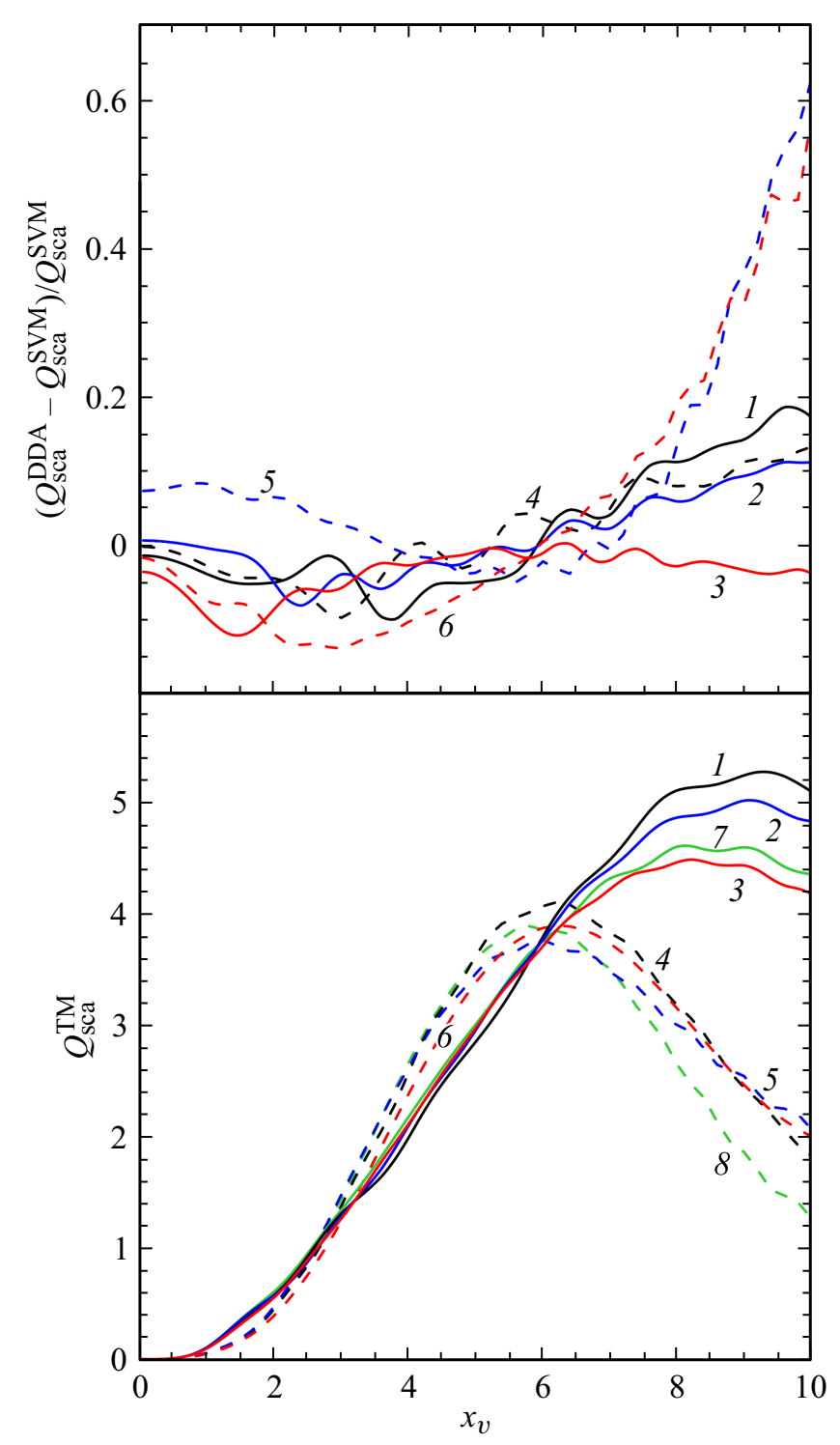

Рис. 4. То же, что и на рис. 1 , но для угла падения $\alpha=90^{\circ}$ и ТМ-моды.

рисунке представлены графики сечений рассеяния сфероидов $Q_{\mathrm{sca}}^{\mathrm{SVM}}$ и частиц иных видов (параллелепипедов, круговых цилиндров и конусов) $Q_{\mathrm{sca}}^{\mathrm{DDA}}$, а также относительные погрешности сфероидальной модели $\delta$ в зависимости от дифракционного параметра $x_{v}$. Отличие рисунков друг от друга заключается в ориентации и поляризации падающего излучения. Первые два рисунка соответствуют падению плоской волны параллельно оси $z$, четыре последних - перпендикулярному падению плоской волны ТЕ- или ТМ-типов.

Обзор полученных результатов показывает, что область применимости модели для параллелепипедов, цилиндров и конусов достаточно широкая для разных параметров задачи. Так, если объемный параметр $x_{v} \leq 6$, то относительная погрешность модели $\delta$ не превышает 10-15\%. При более тщательном анализе видно, что, как 
и ожидалось, первый максимум зависимости факторов рассеяния $Q_{\text {sca }}$ от параметра $x_{v}$ примерно одинаков для рассмотренных частиц, включая модельный сфероид. Очевидно, что область применимости модели связана с этим фактом, и граница области применимости расположена после максимума.

Таким образом, на основании выполненного анализа сфероидальной модели можно сделать выводы: вопервых, о ее применимости для несферических рассеивателей достаточно большого размера $x_{v} \leq 6-8$, и, во-вторых, для частиц, форма которых существенно отличается от сфероидальной (присутствием ребер, вершины и т. п.). При этом модель дает оценку фактора эффективности рассеяния с погрешностью не больше 10-15\%. Отчасти эта погрешность связана с „рябью“ на кривых $Q_{\text {sca }}\left(x_{v}\right)$, имеющей место при вещественной части показателя преломления $n \leq 1.3$ и мнимой части

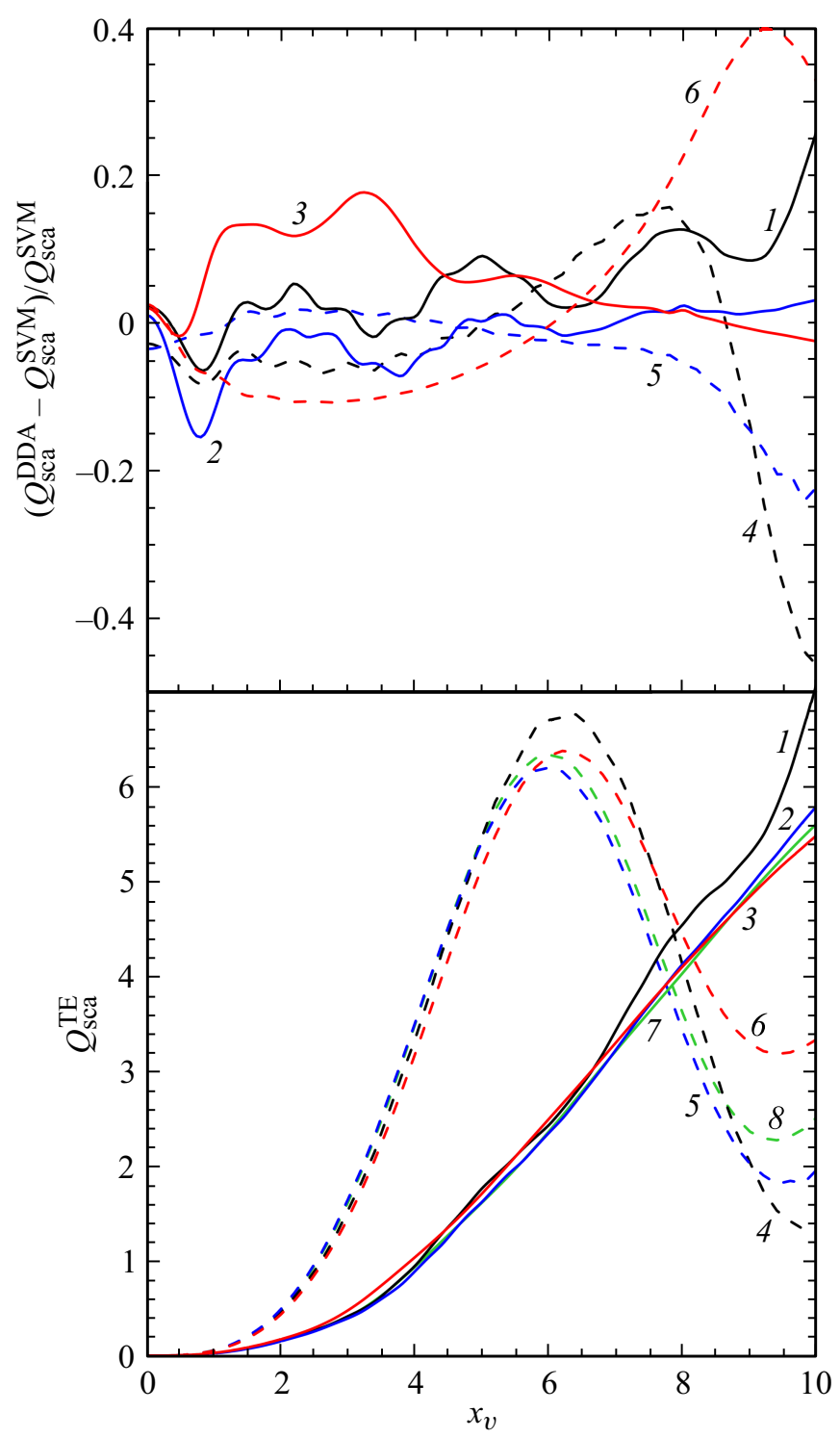

Pис. 5. То же, что и на рис. 2 , но для угла падения $\alpha=90^{\circ}$ и ТЕ-моды.

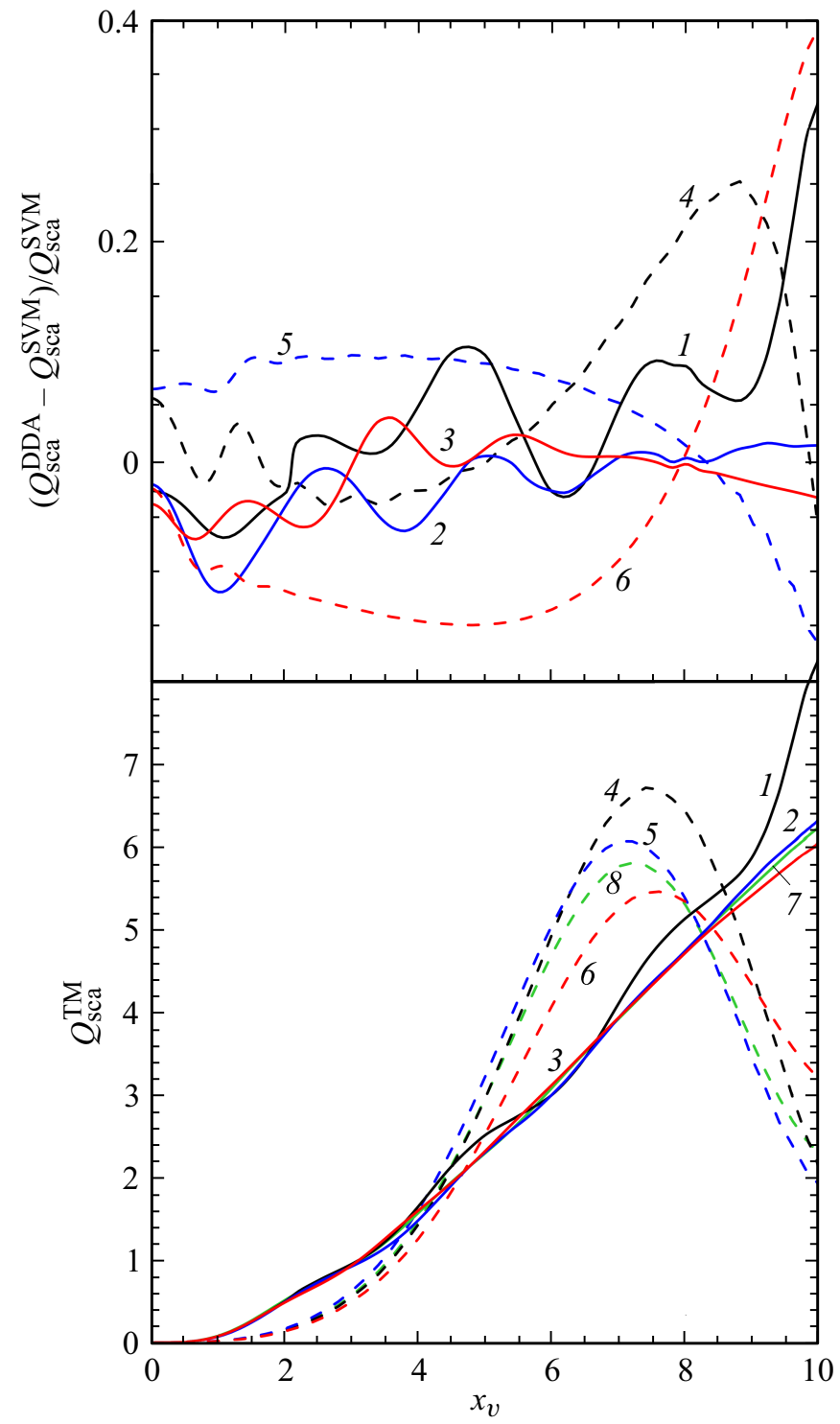

Рис. 6. То же, что и на рис. 2 , но для угла падения $\alpha=90^{\circ}$ и ТМ-моды.

$k=0$. Поэтому для диэлектрических частиц с большим $n$ или $k$ точность модели должна быть несколько лучше.

Заметим, что в работах $[15,16]$ эллипсоидальная модель применялась к малым несфероидальным частицам с использованием приближения Релея и квазистатической аппроксимации. Как оказалось, некоторые трудности с конусами в этих работах были связаны с ошибками применения метода DDA. Исправление этих ошибок показало, что и для конусов предложенная модель вполне пригодна.

Работа была поддержана в 2018-2019 гг. грантом ГУАП и грантами РФФИ 16-02-00194 и 18-52-52006. Авторы благодарны М. Юркину за возможность использовать программу ADDA. 


\section{Список литературы}

[1] Борен К., Хафббмен Д. Поглощение и рассеяние света малыми частицами. М.: Мир, 1986. 660 с.

[2] Mishchenko M.I., Hovenier J.W., Travis L.D. Light Scattering by Nonspherical Particles. San Diego: Academic Press, 2000. $690 \mathrm{p}$.

[3] Mishchenko M.I. Electromagnetic Scattering by Particles and Particle Groups. Cambridge: Cambridge Univ. Press, 2014. $435 \mathrm{p}$.

[4] Rother T., Kahnert M. Electromagnetic Wave Scattering on Nonspherical Particles. Berlin: Springer, 2014. 360 p.

[5] Stegmann P.G. Light Scattering by Non-Spherical Particles. Darmstadt: Techn. Univ. Darmstadt, 2016. 172 p.

[6] Mishchenko M.I., Zakharova T.N., Khlebtsov N.G., Videen G., Wriedt T. // J. Quant. Spectrosc. Rad. Transf. 2017. V. 202. P. 240.

[7] Yurkin M.A., Mishchenko M.I. // Phys. Rev. A. 2018. V. 97. P. 043824.

[8] Dubovik O., Sinyuk A., Lapyonok T., Holben B.N., Mishchenko M. et al. // J. Geophys. Res. Atmosph. 2006. V. 111. P. D11208.

[9] Merikallio S., Lindqvist H., Nousiainen T., Kahnert M. // Atmosph. Chem. Phys. 2011. V. 11. P. 5347.

[10] Liu L., Mishchenko M.I. // J. Quant. Spectrosc. Rad. Transf. 2006. V. 101. P. 488.

[11] Matrosov S.Y. // J. Atmosph. Ocean Techn. 2015. V. 32. P. 865.

[12] Nousiainen T., Kahnert M., Lindqvista H. // J. Quant. Spectrosc. Rad. Transf. 2011. V. 112. P. 2213.

[13] Min M., Hovenier J.W., de Koter A. // Astron. Astrophys. 2003. V. 404. P. 35.

[14] Sihvola A. // J. Nanomater. 2007. V. 2007. P. 45090.

[15] Фарафонов В.Г., Ильин В.Б., Устимов В.И., Тулегенов А.Р. // Опт. и спектр. 2017. Т. 122. № 3. С. 506.

[16] Фарафонов В.Г., Устимов В.И., Прокопьева М.С., Тулегенов А.Р., Ильин В.Б. // Опт. и спектр. 2018. Т. 125. № 6. C. 800 .

[17] Asano S., Yamamoto G. // Appl. Opt. 1975. V. 14, P. 29.

[18] Фарабонов В.Г. // Дифференц. уравн. 1983. Т. 19. С. 1765.

[19] Voshchinnikov N.V., Farafonov V.G. // Astrophys. Space Sci. 1993. V. 204. P. 19.

[20] Purcell E.M., Pennypacker C.R. // Astrophys. J. 1973. V. 186. P. 705.

[21] Draine B.T. // Astrophys. J. 1988. V. 333. P. 848.

[22] Draine B.T., Flatau P. // J. Opt. Soc. Am. A. 1994. V. 11. P. 1491.

[23] Yurkin M.A., Hoekstra A.G. // J. Quant. Spectrosc. Rad. Transf. 2011. V. 112. P. 2234. 